\title{
Left Lung, Superior Lobe, Inferior Lingular Segment
}

National Cancer Institute

\section{Source}

National Cancer Institute. Left Lung, Superior Lobe, Inferior Lingular Segment. NCI

Thesaurus. Code C132400.

The inferior lingual segment of the superior lobe of the left lung. 